\title{
Graph homology and graph configuration spaces
}

\author{
Vladimir Baranovsky • Radmila Sazdanovic
}

Received: 31 July 2011 / Accepted: 23 March 2012 / Published online: 24 April 2012

(C) Tbilisi Centre for Mathematical Sciences 2012

\begin{abstract}
If $R$ is a commutative ring, $M$ a compact $R$-oriented manifold and $G$ a finite graph without loops or multiple edges, we consider the graph configuration space $M^{G}$ and a Bendersky-Gitler type spectral sequence converging to the homology $H_{*}\left(M^{G}, R\right)$. We show that its $E_{1}$ term is given by the graph cohomology complex $C_{A}(G)$ of the graded commutative algebra $A=H^{*}(M, R)$ and its higher differentials are obtained from the Massey products of $A$, as conjectured by Bendersky and Gitler for the case of a complete graph $G$. Similar results apply to the spectral sequence constructed from an arbitrary finite graph $G$ and a graded commutative DG algebra $\mathcal{A}$.
\end{abstract}

Keywords Configuration spaces $\cdot$ Spectral sequence $\cdot$ Homological perturbation

\section{Introduction}

Let $A$ be a graded algebra over a commutative Noetherian ring $R$ of finite $E x t$ dimension. We assume that $A$ is a projective $R$-module.

For any finite graph $G$, we will define the graph cohomology complex $C_{A}(G)$, inspired by the construction of Helme-Guizon and Rong, see for instance [8] or

Communicated by Jim Stasheff.

V. Baranovsky $(\square)$

Department of Mathematics, University of California Irvine,

340 Rowland Hall, Irvine, CA 92617, USA

e-mail:vbaranov@uci.edu

R. Sazdanovic

Department of Mathematics, University of Pennsylvania,

209 South 33rd Street, Philadelphia, PA 19104-6395, USA

e-mail: radmilas@math.upenn.edu 
Section 2 of [7]. To that end, let $E(G)$ and $V(G)$ be the sets of edges and vertices, respectively, and choose a bijection of $V(G)$ with $\{1, \ldots, n\}$, i.e. an enumeration of the vertices. This gives an orientation for any edge $\alpha \in E(G)$ : if $\alpha$ connects vertices $i$ and $j$ with $i \leq j$ we write $\alpha: i \rightarrow j$. For any subset $s \subset E(G)$ let $l(s)$ be the number of connected components in the subgraph $[G: s]$ which has the same set of vertices as $G$ but the edges in $s$ only.

Denote by $\Lambda=\Lambda\left(e_{\alpha}\right)$ the exterior algebra over $R$ on the generators $e_{\alpha}, \alpha \in E(G)$. For $s \subset E(G)$ set $e_{s}$ to be the exterior product of all $e_{\alpha}, \alpha \in s$, ordered with respect to the lexicographic ordering on the pairs $(i, j)$ coming from the edges $\alpha: i \rightarrow j$. Similarly, the connected components of $[G: s]$ are naturally ordered by the smallest vertex contained in a component.

Now define the bigraded complex $C_{A}(G)$ to be the quotient algebra of $\Lambda \otimes_{R} A^{\otimes n}$ by the relations, cf. [2, p. 428]:

$$
e_{\alpha} \otimes(a[i]-a[j]), \quad a \in A, E(G) \ni \alpha: i \rightarrow j
$$

where we denote by $a[i]$ the element $1^{\otimes(i-1)} \otimes a \otimes 1^{\otimes(n-i)} \in A^{\otimes n}$ for $i \in\{1, \ldots, n\}$.

The complex $C_{A}(G)$ has a natural bigrading in which each $e_{\alpha}$ has bidegree $(0,1)$, and $a_{1} \otimes \cdots \otimes a_{n} \otimes 1$ has bidegree $\left(\sum_{i=1}^{n} \operatorname{deg}_{A}\left(a_{i}\right), 0\right)$. The differential $\delta$ on $C_{A}(G)$ of bidegree $(0,1)$ is given by the wedge product with $\sum_{\alpha \in E(G)} e_{\alpha}$.

Alternatively, we can define $C_{A}(G)$ in terms of subgraphs in $E(G)$ as the complex of projective $R$-modules

$$
C_{A}(G):=\bigoplus_{s \subset E(G)} e_{s} \cdot A^{\otimes l(s)},
$$

where $e_{s} \cdot a_{1} \otimes \cdots \otimes a_{l(s)}$ has bidegree $\left(\sum_{i=1}^{l(s)} \operatorname{deg}_{A} a_{i},|s|\right)$, with $\delta$ acting as follows

$$
\begin{aligned}
& \delta\left(e_{s} \cdot a_{1} \otimes \cdots \otimes a_{l(s)}\right)=\sum_{\substack{\alpha \in E(G) \\
l(s \cup \alpha)=l(s)}} e_{\alpha} e_{s} \cdot a_{1} \otimes \cdots \otimes a_{l(s)} \\
& +\sum_{\substack{\alpha \in E(G) \\
l(s \cup \alpha)=l(s)-1}}(-1)^{\tau} e_{\alpha} e_{s} \cdot a_{1} \otimes \cdots \otimes a_{t(\alpha)} a_{h(\alpha)} \otimes \cdots \otimes a_{l(s)}
\end{aligned}
$$

where $s \cup \alpha$ is the subset obtained by adding $\alpha: i \rightarrow j$ to $s$ (we can assume that $\alpha \notin s$ as otherwise $\left.e_{s} e_{\alpha}=0\right)$, and $t(\alpha)$ and $h(\alpha)$ are the numbers of the connected components in the subgraph $[G: s]$ containing $i$ and $j$, respectively. The first sum corresponds to the case $h(\alpha)=t(\alpha)$ and the second to $h(\alpha) \neq t(\alpha)$. Note that $i$ and $j$ may not be the smallest vertices in their connected components and one can have $h(\alpha)<t(\alpha)$ or $t(\alpha)<h(\alpha)$. Depending on that, the product $a_{t(\alpha)} a_{h(\alpha)}$ has either $(t(\alpha)-2)$ or $(t(\alpha)-1)$ terms to the left of it. The sign $(-1)^{\tau}$ in the second group of terms is the Koszul sign of the permutation of $a_{1}, \ldots, a_{l(s)}$ which moves $a_{h(\alpha)}$ to the immediate right of $a_{t(\alpha)}$, and preserves the order of other elements. 
Now let $M$ be a simplicial complex. For any $\alpha: i \rightarrow j$ let $Z_{\alpha}$ be the diagonal in the cartesian product $M^{n}$ defined by $m_{i}=m_{j}$, and set

$$
Z_{G}=\bigcup_{\alpha \in E(G)} Z_{\alpha}, \quad M^{G}=M^{n} \backslash Z_{G} .
$$

We will call $M^{G}$ the graph configuration space of $M$. In the case when $G$ is the complete graph on $n$ vertices we get the classical configuration spaces of ordered $n$-tuples of pairwise distinct points in $M$.

Theorem 1 Assume that the cohomology algebra $A=H^{*}(M, R)$ is a projective $R$-module and that $G$ has no loops or multiple edges. There exists a spectral sequence with $E_{1}$ term isomorphic to $C_{G}(A)$ which converges to the relative cohomology $H^{*}\left(M^{\times n}, Z_{G} ; R\right)$.

Remark 2 This theorem resolves the conjecture of M. Khovanov, that there exists a spectral sequence from chromatic graph cohomology defined by Helme-Guizon and Rong [8] to Eastwood-Hugget graph homology [4]. A standard consequence of the theorem is equality of the Poincare polynomials (with respect to the total grading) for $C_{A}(G)$ and $H^{*}\left(M^{\times n}, Z_{G} ; R\right)$.

Remark 3 For a general graph $G$ and an edge $\alpha \in E(G)$ one can use the deletioncontraction sequence

$$
0 \rightarrow C_{A}(G / \alpha) \rightarrow C_{A}(G) \rightarrow C_{A}(G \backslash \alpha) \rightarrow 0
$$

to compute the graph cohomology. There $G / \alpha$ is the graph obtained by contracting $\alpha$ to a single vertex, and $G \backslash \alpha$ is obtained by removing $\alpha$. Then it is easy to see that the graph cohomology is zero when $G$ has a loop, and it does not change if multiple edges $i \rightarrow j$ get replaced by a single edge.

Remark 4 When $M$ is a compact $R$-orientable manifold of dimension $m$, the relative cohomology groups $H^{*}\left(M^{\times n}, Z_{G} ; R\right)$ are isomorphic to the homology groups $H_{m n-*}\left(M^{G} ; R\right)$ by Lefschetz duality. Observe, however, that for existence of the spectral sequence we still have to assume that $H^{*}(M, R)$ is projective over $R$ (one of the reasons is that we use the Kunneth formula for cohomology). In general the cohomology algebra $A$ needs to be replaced by an appropriate projective DG-algebra $\mathcal{A}$ resolving it, and $C_{A}(G)$ by $C_{\mathcal{A}}(G)$, as in Sect. 2.2 below.

In Sect. 4 we study the higher differentials of this spectral sequence and show that they are determined by the matrix Massey products of $A$, as conjectured by Bendersky and Gitler [2]. Our main results here are Proposition 14, explaining how the $A_{\infty}$-algebra structure on $A$ and an application of perturbation theory to spectral sequences (as recalled in Proposition 11) allow us to compute the spectral sequence differentials; and Proposition 18 which says that the spectral sequence degenerates starting with the page $E_{m}$, where $m$ is the number of vertices in the largest subtree of $G$. Also, a standard argument shows that in some cases, e.g. when $M$ is a compact Kähler manifold, the spectral sequence degenerates in the $E_{2}$ term. 


\section{Spectral sequences}

\subsection{Proof of Theorem 1}

For any simplicial topological space $X$, we denote by $C^{*}(X ; R)$ its cochain complex. Suppose that $Z \subset X$ is a subspace which is a union of closed subspaces $Z_{\alpha}, \alpha \in E$, where $E$ is a finite ordered set. For a finite subset $s \subset E$ let

$$
Z_{s}=\bigcup_{\alpha \in s} Z_{\alpha}
$$

and denote $Z_{\emptyset}=X$ for notational convenience. By pages 425 and 427 of [2], the relative cohomology $H^{*}(X, Z ; R)$ can be computed as the total cohomology of a bicomplex

$$
C^{*}\left(Z_{\emptyset}, R\right) \rightarrow \bigoplus_{\alpha \in E} C^{*}\left(Z_{\alpha} ; R\right) \rightarrow \bigoplus_{s \subset E ;|s|=2} C^{*}\left(Z_{s} ; R\right) \rightarrow \ldots
$$

where the differential comes from the obvious simplicial structure on the collection of subsets in $E$. Applying one of the two standard spectral sequences of a bicomplex we obtain a spectral sequence converging to $H^{*}(X, Z ; R)$ with

$$
E_{1}^{p q}=\bigoplus_{s \subset E ;|s|=p} H^{q}\left(Z_{s} ; R\right)
$$

and the differential $\partial_{1}: E_{1}^{p, q} \rightarrow E_{1}^{p+1, q}$ is the usual simplicial differential constructed from the pullbacks with respect to the closed embeddings

$$
Z_{t}=Z_{s} \cap Z_{\alpha} \subset Z_{s} ; \quad t=s \cup\{\alpha\}, \alpha \notin s .
$$

Next, we specialize to the case when $X=M^{n}, G$ has no loops or multiple edges and $Z=\bigcup_{\alpha \in E(G)} Z_{e}$ comes from the set $E=E(G)$ of edges in $G$ (any multiple edges would not be reflected in the geometric construction and a loop would lead to $Z=X$ ). For a general subset $s \subset E$ the space $Z_{s}$ can be identified with $M^{l(s)}$. By projectivity (and hence flatness) of $A$, the Kunneth formula applies to give

$$
H^{*}\left(Z_{s}, R\right)=A^{\otimes l(s)}
$$

where the tensor product is taken over $R$.

To compute the differentials explicitly, consider two cases. Firstly, the unique element $\alpha \in t \backslash s$ may connect two vertices within the same connected component of $[G: s]$. In this case the embedding $Z_{t} \subset Z_{s}$ is an isomorphism and hence induces the identity map on cohomology.

Secondly, $\alpha$ may connect two of the $l=l(s)$ connected components of the graph $[G: s]$. To simplify notation assume that these are the components corresponding to 
the first two factors of $A^{\otimes l}$. Then the embedding $Z_{t} \rightarrow Z_{s}$ is the product of the diagonal map $M \rightarrow M \times M$ and the identity on the other $M^{l-2}$ factors. But the pullback with respect to the diagonal map induces on cohomology precisely the cup product $A \otimes A \rightarrow A$. Hence $Z_{t} \subset Z_{S}$ in this case induces the map

$$
A^{\otimes l} \rightarrow A^{\otimes(l-1)}, \quad a_{1} \otimes a_{2} \otimes a_{2} \otimes \cdots \otimes a_{l} \mapsto a_{1} a_{2} \otimes a_{3} \otimes \cdots \otimes a_{l} .
$$

This finishes the proof of the theorem.

Remark 5 One can give a slight generalization when there is a continuous map $f$ : $N \rightarrow M$ which makes $B=H^{*}(N, R)$ into a module over $A=H^{*}(M, R)$. In this case we assume that the set of vertices in the graph $G$ is $I=\{0, \ldots, n\}$ and define the generalized configuration space $M^{G, f}$ as the open complement $\left(N \times M^{\times n}\right) \backslash Z_{G}$. When the edge $\alpha$ connects two nonzero vertices the subset $Z_{\alpha}$ is still defined by the condition $m_{i}=m_{j}$ while for an edge connecting $i=0$ and $j$ we use the condition $f(n)=m_{j}$. As with $A$, we need to assume that $B$ is projective over $R$.

Since the graph embedding $\left(I d_{N}, f\right): N \rightarrow N \times M$ induces the module action map $B \otimes_{R} A \rightarrow B$ on cohomology, we get the spectral sequence with $E_{1}$ given by the graph cohomology of the pair $(A, B)$ (the complex is constructed similarly, but the $A$-module $B$ is placed at the zero vertex), converging to the relative cohomology $H^{*}\left(N \times M^{n}, Z_{G} ; R\right)$. When $M, N$ are compact $R$-orientable manifolds and $f$ is a smooth map, this is isomorphic to the homology of the generalized configuration space $M^{G, f}$.

\subsection{Graph cohomology of DG algebras}

Let $\mathcal{A}$ be a commutative DG algebra. Then the complex $C_{\mathcal{A}}(G)$ has another differential $d$ of bidegree $(1,0)$ induced by the differential of $\mathcal{A}$, and it is easy to check that $d \delta+\delta d=0, d^{2}=0$. Therefore we can consider the total differential $D=d+\delta$.

Now assume that $\mathcal{A}$ is a commutative DG algebra such that the bicomplex $C_{\mathcal{A}}(G)$ may be connected with the graph configuration space bicomplex (1) by a sequence of morphisms of first quadrant bicomplexes, each inducing quasi-isomorphisms along the columns. Then the two bicomplexes have isomorphic spectral sequences (starting with the $E_{1}$ term) associated with the vertical filtration, as follows from the standard definitions e.g. in [6].

Example 6 We can take $\mathcal{A}=H^{*}(M ; R)$ with the zero differential if the space $M$ is $R$-formal, i.e. if $H^{*}(M ; R)$ and the cochain algebra $C^{*}(M ; R)$ may be connected with $H^{*}(M ; R)$ by a chain of DG algebra quasi-isomorphisms. When $R$ is the field $\mathbb{Q}$ of rational numbers, we can take the complex of Sullivan cochains, and for the field $\mathbb{R}$ of real numbers we can take the De Rham complex of differential forms. Finally by a result of [1], for $R$ a field of finite characteristic $p$, the DG algebra $\mathcal{A}$ exists if $M$ is $r$-connected and $p r>\operatorname{dim} M$.

Remark 7 When the algebra $A$ is not flat over $R$, a better version of its graph homology is obtained by taking a flat $D G$-resolution $\mathcal{A} \rightarrow A$ and then computing the cohomology of $C_{\mathcal{A}}(G)$ with respect to the total differential. 


\section{Perturbation Lemma and applications}

The material of this section is fairly standard, we collect it here for the reader's convenience and also to fix the notation.

\subsection{Basic Perturbation Lemma}

Definition 8 Let $K, L$ be a pair of complexes with differentials $d_{K}, d_{L}$ respectively. Consider morphisms of complexes $f: K \rightarrow L, g: L \rightarrow K$ such that $f g$ is equal to the identity $1_{L}$ on $L$ and $1_{K}-g f=d_{K} h+h d_{K}$ for some homotopy $h$. The triple $(f, g, h)$ is called a reduction (or a strong deformation retract) if in addition the following side conditions are satisfied

$$
h g=f h=h h=0 .
$$

It is well known, cf. [9], that conditions (2) can be ensured by adjusting an arbitrary homotopy $h$ : first replacing it by $h^{\prime}=(d h+h d) h(d h+h d)$ which satisfies $h^{\prime} g=f h^{\prime}=0$, and then further setting $h^{\prime \prime}=h^{\prime} d h^{\prime}$ which will imply all three side conditions.

Remark 9 Suppose that $K$ is a complex of projective $R$-modules, such that $L=$ $H^{*}(K)$ is also projective, and that the commutative ring $R$ has finite $E x t$ dimension. Denoting by $B^{n}$, resp. $Z^{n}$ the coboundaries, resp. the cocycles, of $K$, we have the standard exact sequences:

$$
0 \rightarrow B^{n} \rightarrow Z^{n} \rightarrow L^{n} \rightarrow 0 ; \quad 0 \rightarrow Z^{n} \rightarrow K^{n} \rightarrow B^{n+1} \rightarrow 0 .
$$

Since we assumed $L^{n}$ and $K^{n}$ to be projective, this gives for any $R$-module $M$ the isomorphisms for $i \geq 1$ and all $n$ :

$$
\operatorname{Ext}_{R}^{i}\left(Z^{n}, M\right) \simeq \operatorname{Ext}_{R}^{i}\left(B^{n}, M\right) ; \quad \operatorname{Ext}_{R}^{i}\left(Z^{n}, M\right)=\operatorname{Ext}_{R}^{i+1}\left(B^{n+1}, M\right) .
$$

Since we assumed $R$ to be of finite $E x t$-dimension, we get by induction on $k$ that $\operatorname{Ext}_{R}^{i}\left(B^{n}, M\right)=\operatorname{Ext}_{R}^{i+k}\left(B^{n+k}, M\right)$ which must be zero if $k$ is large enough. Therefore each $B^{n}$ is also projective and we can choose splittings

$$
K^{n} \simeq B^{n+1} \oplus Z^{n} \simeq B^{n+1} \oplus B^{n} \oplus L^{n}
$$

such that the differential $K^{n} \rightarrow K^{n+1}$ is the composition of the projection $K^{n} \rightarrow$ $B^{n+1}$ and the embedding $B^{n+1} \rightarrow K^{n+1}$. Then a reduction $(f, g, h)$ may be defined as follows: $f, g$ are the obvious projection and embedding and $h$ is the "inverse" composition $K^{n+1} \rightarrow B^{n+1} \rightarrow K^{n}$.

Now suppose we have a perturbation $\widehat{d}_{K}=d_{K}+\delta_{K}$ of the differential $d_{K}$ such that $\delta_{K}^{2}=0, d_{K} \delta_{K}+\delta_{K} d_{K}=0$. We assume in addition that the composition $\delta_{K} H$ 
is locally nilpotent, i.e. on any particular $x \in K$ we have $\left(\delta_{K} h\right)^{n} x=0$ where the positive integer $n$ may depend on $x$.

The following result is known as the Basic Perturbation Lemma, see [9] and references in that paper.

Lemma 10 Under the above assumptions, there exist: a perturbation of the differential $\widehat{d}_{L}=d_{L}+\delta_{L}$ on L, morphisms of complexes $\widehat{f}: K \rightarrow L, \widehat{g}: L \rightarrow K$ and a homotopy $\widehat{h}$ (with respect to the perturbed differentials on $K, L$ ), given by the formulas

$$
\delta_{L}=f X g, \widehat{f}=f(1-X h), \widehat{g}=(1-h X) g, \widehat{h}=h-h X h ;
$$

where

$$
X=\delta_{K}-\delta_{K} h \delta_{K}+\left(\delta_{K} h\right)^{2} \delta_{K}-\left(\delta_{K} h\right)^{3} \delta_{K}+\ldots
$$

\subsection{Perturbations and spectral sequences}

Now we apply the previous result to give a very concrete realization of the spectral sequence of a bicomplex of modules over a ring, in the case when a reduction is chosen for one of the differentials (say the vertical). Although it is not easy to find an exposition of this approach in the published literature (but see [15], for instance) it is fairly old and known to the experts in the field.

Consider a bicomplex with a vertical $d: A^{p, q} \rightarrow A^{p, q+1}$ and a horizontal differential $\delta: A^{p, q} \rightarrow A^{p+1, q}$. We want to identify the higher differentials of the standard spectral sequence with $E_{1}^{p, q}=H_{d}\left(A^{p, q}\right)$ converging to the cohomology of the total complex $(K, d+\delta)$.

To that end, let $L$ be the total complex of the $E_{1}$ term and assume there is a reduction of $A^{p, \cdot}$ to $H_{d}\left(A^{p, \cdot}\right)$ along each column of the original bicomplex. This induces a reduction $(f, g, h)$ of the total complex $(K, d)$ onto $(L, 0)$.

Trying to compute the cohomology of $(K, d+\delta)$, we can treat $d+\delta$ as a perturbation of $d$ and apply the Basic Perturbation Lemma 10. Observe that the local nilpotence condition on $\delta h$ holds, for example, when $A^{p, q}$ is concentrated in the first quadrant (since $\delta$ moves an element to the right, and $h$ moves it down).

By the Basic Perturbation Lemma, the complexes $(K, d+\delta)$ and $(L, f X g)$ are homotopic; hence instead we can compute the cohomology of $L$ with respect to

$$
\widehat{d}_{L}=d_{1}+d_{2}+d_{3}+d_{4}+\ldots
$$

where

$$
d_{i}=(-1)^{i-1} f(\delta h)^{i-1} \delta g
$$

Each $d_{i}$ is an operator $E_{1}^{p, q} \rightarrow E_{1}^{p+i, q+1-i}$.

Since the homotopy $h$ preserves the filtration on $K$ the spectral sequences of filtered complexes $K$ and $L$ agree, see e.g. Theorem 15 in [15]. Writing out the standard 
definitions for the spectral sequence of the filtered complex $(L, f X g)$ we get the following result.

Proposition 11 For every $i \geq 2$ an element of $E_{i}^{p, q}$ is represented by $x \in L^{p, q}$ such that the following system of equations on $x_{2}, \ldots, x_{i-1}$ admits a solution

$$
\begin{array}{cl}
d_{1}(x)=0 ; \quad & d_{2}(x)+d_{1}\left(x_{2}\right)=0 ; \quad d_{3}(x)+d_{2}\left(x_{2}\right)+d_{1}\left(x_{3}\right)=0 ; \ldots \\
& d_{i-1}(x)+d_{i-2}\left(x_{2}\right)+\cdots+d_{1}\left(x_{i-1}\right)=0,
\end{array}
$$

modulo the elements of the form $x=d_{i-1}\left(b_{2}\right)+\cdots+d_{2}\left(b_{i-1}\right)+d_{1}\left(b_{i}\right)$ where $b_{i}$ is arbitrary, and $\left(b_{2}, \ldots, b_{i-2}\right)$ satisfy a system of equations, obtained from (4) by setting $x=0$ and replacing $x_{j}$ by $b_{j}$. The value of the differential $\partial_{i}: E_{i}^{p, q} \rightarrow E_{i}^{p+i, q+1-i}$ on such $x$ is represented by the following element of $E_{1}^{p+i, q+1-i}$ :

$$
d_{i}(x)+d_{i-1}\left(x_{2}\right)+\cdots+d_{2}\left(x_{i-1}\right) .
$$

Corollary 12 Let $i \geq 2$ and suppose that $x \in E_{1}^{p, q}$ is such that $d_{1}(x)=d_{2}(x)=$ $\cdots=d_{i-1}(x)=0$. Then such $x$ represents a class in $E_{i}^{p, q}$ (as we may simply take $x_{i}=0$ for $i \geq 2$ ) and $\partial_{i}(x)$ is represented by $d_{i}(x) \in E_{1}^{p+i, q-i+1}$.

\subsection{A-infinity structures on cohomology and Massey products}

Let $\mathcal{A}$ be a DG algebra and $A$ its cohomology algebra and assume there is a reduction $(f, g, h)$ of $\mathcal{A}$ to $A$ as before. Note that in general it may not be possible to choose either $f$ or $g$ multiplicative (e.g. if the derived categories of $\mathcal{A}$ and $A$ are not equivalent). In fact, $A$ admits a system of products $m_{k}: A^{\otimes k} \rightarrow A, i \geq 2$, which, in a sense, measure how far the two algebras are from being quasi-isomorphic as DG algebras, cf. [10].

We recall this construction from the perturbation theory viewpoint. First consider $\mathcal{A}$ and $A$ as non-unital algebras with the zero product and consider their bar constructions $B(\mathcal{A})=\bigoplus_{k \geq 0} \mathcal{A}^{\otimes k}$ and similarly for $B(A)$. The differential on $\mathcal{A}$ extends by the Leibnitz rule to $B(\mathcal{A})$ and the original contraction $(f, g, h)$ extends to a contraction from $B(\mathcal{A})$ to $B(A)$. The extension for $f$ and $g$ is obvious, and $h$ is given in $A^{\otimes n}$ by

$$
\sum_{i=1}^{n}(g f)^{\otimes(i-1)} \otimes h \otimes 1^{\otimes(n-i)}
$$

If we now recall the non-trivial product on $\mathcal{A}$, this will give a perturbation $d+\delta$ of the initial differential $d$ on $K=B(\mathcal{A})$. Hence by Perturbation Lemma 10 we can write a new non-zero differential on $L=B(A)$ such that the two bar constructions are still homotopic. One can check that the new differential agrees with the natural coproduct on $B(A)$ and it is therefore encoded by a series of maps $m_{k}: A^{\otimes k} \rightarrow A$. One further checks that $m_{1}=0$ and $m_{2}$ is the standard product $f(g(a) g(b))$. 
Explicitly, one can define $m_{n}: A^{\otimes n} \rightarrow A$ using the operations $\lambda_{n}: \mathcal{A}^{\otimes n} \rightarrow \mathcal{A}$ for $n \geq 2$ by setting $\lambda_{2}\left(a_{1} \otimes a_{2}\right)=a_{1} a_{2}$ and

$$
\lambda_{n}=\sum_{p=1}^{n-1}(-1)^{p+1} \lambda_{2}\left[h \lambda_{p} \otimes h \lambda_{n-p}\right]
$$

where in the terms with $p=1$ and $n-1$ we formally set $h \lambda_{1}=-i d_{\mathcal{A}}$. Then

$$
f \circ \lambda_{n} \circ g^{\otimes n}: A^{\otimes n} \rightarrow A
$$

for $n \geq 2$ gives an $A_{\infty}$-structure $\left\{m_{n}\right\}_{n \geq 2}$. See $[3,9,12]$ and references therein.

The higher products $m_{k}$ for $k \geq 3$ in general depend on the choice of $h$. However, for special choices of $a_{1}, \ldots, a_{k}$ the value $m_{k}\left(a_{1}, \ldots, a_{k}\right)$ belongs to the coset of a Massey product, see Theorem 3.1 in [12], see also Theorems 6.3 and 6.4 in [9], hence at least this coset is independent of $h$ for this particular choice of the arguments.

\section{Higher differentials and Massey products}

As in the previous subsection, any reduction $(f, g, h)$ of $\mathcal{A}$ to $A$ induces a reduction of the graph cohomology complex $C_{G}(\mathcal{A})$ onto $C_{G}(A)$. We will show that the operators $d_{i}$ defined by (3) are completely determined by the $A_{\infty}$-structure on $A$, induced by $(f, g, h)$. In view of Proposition 11, this gives information about the higher differentials of the spectral sequence. In addition, specific values of the $A_{\infty}$ operations are given by Massey products, see below, confirming the conjecture by Bendersky and Gitler (formulated originally for the complete graph $G$, i.e. the usual configuration space).

For other situations in which differentials of a spectral sequence are related to the (matric) Massey products see Theorem 12.1 in [16], Corollary 4.6 in [13] or [11].

\subsection{Computation of the $d_{i}$ operators}

We want to compute the values of $d_{i}: E_{1}^{p, q} \rightarrow E_{1}^{p+i, q+1-i}$. Write

$$
d_{i}\left(e_{s} \cdot a_{1} \otimes \cdots \otimes a_{l(s)}\right)=\sum_{t \mid s \subset t} e_{t} \cdot C_{i}^{s \subset t}\left(a_{1} \otimes \cdots \otimes a_{l(s)}\right)
$$

Proposition 13 The value of $C_{i}^{s \subset t}\left(a_{1} \otimes \cdots \otimes a_{l(s)}\right)$ is zero unless the set of edges $t \backslash s$ has $i$ elements and projects to a tree with $i$ edges in the graph $G / s$ obtained by contracting all edges in $s$. In the latter case, suppose that the edges of $t \backslash s$ connect the components $1 \leq \alpha_{1}<\alpha_{2}<\cdots<\alpha_{i+1} \leq l(s)$ in the graph $[G: s]$, then

$$
\begin{gathered}
C_{i}^{s \subset t}\left(a_{1} \otimes \cdots \otimes a_{l(s)}\right)=(-1)^{\varepsilon} a_{1} \otimes \cdots \otimes a_{\alpha_{1}-1} \otimes \\
\otimes C_{i}^{\emptyset \subset(t \backslash s)}\left(a_{\alpha_{1}} \otimes \cdots \otimes a_{\alpha_{i+1}}\right) \otimes \ldots \widehat{a_{\alpha_{2}}} \ldots \widehat{a_{\alpha_{i+1}}} \otimes \cdots \otimes a_{l(s)},
\end{gathered}
$$


and $(-1)^{\varepsilon}$ is the sign of the permutation

$$
\left(a_{1}, \ldots, a_{l(s)}\right) \mapsto\left(a_{1}, \ldots, a_{\alpha_{1}-1}, a_{\alpha_{1}}, a_{\alpha_{2}}, \ldots, a_{\alpha_{i+1}}, \ldots \widehat{a_{\alpha_{2}}} \ldots \widehat{a_{\alpha_{i+1}}}, \ldots, a_{l(s)}\right) .
$$

Proof The side conditions $h g=h h=0$ imply that when we evaluate $\delta(h \delta)^{i-1}$ on a tensor monomial, all occurrences of $h$ should be applied only to the newly created products involved in the definition of $\delta$. Also, the last occurrence of $h$ should be multiplied by something else before we apply $f$ to it (since $f h=0$ ). Therefore, every tensor factor standing next to $e_{t}$ in $f^{\otimes(l(s)-i)} \delta(h \delta)^{i-1} g^{\otimes l(s)}\left(e_{s} \cdot a_{1} \otimes \ldots a_{l(s)}\right)$, is either one of the original $a_{i}$, or an expression involving $p$ multiplications and $\leq p-1$ uses of $h$. Since the total number of multiplications is $i$ and $h$ occurs $(i-1)$ times, exactly one of those factors can have the latter form. This means that $i$ components of $[G: s]$ assemble into a single component of $[G: t]$ and other components remain untouched, as claimed. The rest follows from the contraction isomorphism of complexes $e_{s} \cdot C_{G}(A) \simeq C_{G / s}(A)$.

The previous proposition means that, in computing operators $d_{i}$ (involved in the formulas for the spectral sequence differentials $\partial_{i}$ ) we can reduce to the case when $s=\emptyset, t=E(G)$, and $G$ is a connected tree with $i=n-1$ edges.

Proposition 14 Under the above assumptions

$$
d_{n-1}\left(a_{1} \otimes \cdots \otimes a_{n}\right)=(-1)^{\frac{n(n-1)}{2}} \sum_{\sigma \in \Sigma(G)}(-1)^{\sigma} e_{t} \cdot m_{n} \circ \sigma
$$

where $m_{n}: A^{\otimes n} \rightarrow A$ is the $n$-th product of the $A_{\infty}$-structure on $A$ induced by the reduction of $\mathcal{A}$ onto $A$ as in (6); and the sum runs over the set $\Sigma(G)$ of all permutations of $\{1, \ldots, n\}$ such that the total order in which $\sigma(1)<\sigma(2)<\cdots<\sigma(n)$ refines the partial order generated by $i<j$ whenever there is an edge $i \rightarrow j$ in the graph $G$.

Note that the action of $\sigma: A^{\otimes n} \rightarrow A^{\otimes n}$ involves an appropriate Koszul sign.

Remark 15 See Theorem 12.1 on page 60 of [16] for a similar (and perhaps related) situation when a spectral sequence differential is related to an $A_{\infty}$ structure. See also [5] for a special case (with $n=3$ ) of the above result.

Example 16 In the example shown on Fig. 1 the possible permutations $\sigma$ are given by

$$
\text { (1234567), (2134567), (1234576), (2134576), (1234756), (2134756). }
$$

Proof We use induction on $n$. For $n=2$ the graph $G$ consists of a single arrow and the assertion easily follows from the definition of $d_{1}$. For general $n$ let us consider the

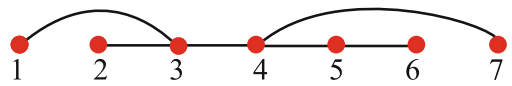

Fig. 1 An example for $n=7$ 
last edge $\alpha$ to disappear when we apply the leftmost term in the expression $\delta(h \delta)^{n-2}$ to the element $g^{\otimes n}\left(a_{1} \otimes \cdots \otimes a_{n}\right)$. When we remove $\alpha: i \rightarrow j$ the graph $G$ splits into a disjoint union of two trees. We have three cases. First, one of this trees may consist of a single vertex $i$. Denoting the other tree by $G_{1}$ we encode this case by the diagram $i \rightarrow G_{1}$. Next, both trees may contain at least two vertices. Denoting these trees by $G_{2}$ and $G_{3}$ we will use the notation $G_{2} \rightarrow G_{3}$. Finally, if one of the trees is the single vertex $j$ and the other is denoted by $G_{4}$ we encode this situation by $G_{4} \rightarrow j$.

Denote by $\lambda_{G}$ the expression $\delta(h \delta)^{n-2}$. We would like to show that $\lambda_{G}$ is equal to the alternating sum of $\lambda_{n} \circ \sigma, \sigma \in \Sigma_{n}$, modulo the image of $h$ (which does not affect the value of $d_{i}$ due to $f h=0$ ). It is clear that looking at "the last edge to be used with $\delta$ " we get an inductive formula

$$
\lambda_{G} \equiv \sum_{i \rightarrow G_{1}} \pm \lambda_{2}\left[h \lambda_{1} \otimes h \lambda_{G_{1}}\right]+\sum_{G_{2} \rightarrow G_{3}} \pm \lambda_{2}\left[h \lambda_{G_{2}} \otimes h \lambda_{G_{3}}\right]+\sum_{G_{4} \rightarrow j} \pm \lambda_{2}\left[h \lambda_{G_{4}} \otimes h \lambda_{1}\right]
$$

modulo terms in the image of $h$. We would like to establish the inductive step by applying the antisymmetrization in $\sigma$ to the formula (5) and comparing the result with the above recursive formula. The first terms matches the $p=1$ term in the antisymmetrization of (5) since the vertices $i$ which can occur in $i \rightarrow G_{1}$ are exactly the vertices which can occur as $\sigma(1)$, and for the second factor we can apply the inductive assumption to $G_{1}$. Similarly the third term above matches the $s=p-1$ term in the antisymmetrization of (5) since the possible values of $\sigma(n)$ are exactly the vertices $j$ which have a single edge coming into it.

Hence it remains to compare the second term above and the terms corresponding to $2 \leq p \leq n-2$ in the formula (5). For the latter terms, consider $\sigma \in \Sigma_{G}$, then we want to understand $h \lambda_{p}\left(a_{\sigma(1)} \otimes \ldots a_{\sigma(p)}\right) \otimes h \lambda_{n-p}\left(a_{\sigma(p+1)} \otimes \cdots \otimes a_{\sigma(n)}\right)$ This appears in the middle term of (7) precisely when both subsets of vertices $\sigma(1), \ldots, \sigma(p)$ and $\sigma(p+1), \ldots, \sigma(n)$ give connected subgraphs $G_{2}$ and $G_{3}$, respectively.

We would like to show that all other terms sum to zero. We group together those terms that give fixed $G_{2}$ and $G_{3}$ and assume that $G_{2}$ has $q \geq 2$ connected components $J_{1}, \ldots, J_{q}$. Observe that the total order induced by $\sigma$ is in this case simply a concatenation of total orders on $G_{2}$ and $G_{3}$, and the total order on $G_{2}$ must refine the partial order induced by the edges of $G$, i.e. it is simply a shuffle of total orders on $J_{1}, \ldots, J_{q}$. Hence in the antisymmetrization the operator $h \lambda_{s}$ is applied to

$$
\pm\left[\sum_{\sigma_{1} \in \Sigma_{J_{1}}}(-1)^{\sigma_{1}} \sigma_{1}\left(a_{J_{1}}\right)\right] \# \ldots \#\left[\sum_{\sigma_{q} \in \Sigma_{J_{q}}}(-1)^{\sigma_{1}} \sigma_{q}\left(a_{J_{q}}\right)\right]
$$

where \# stands for the shuffle product on the tensors and $a_{J_{i}}$ is the ordered tensor product of elements in $J_{i}$. Since $\mathcal{A}$ is graded commutative, the operations $h \lambda_{s}$ vanish when applied to shuffle products by Theorem 12 in [3] (in fact, we use the vanishing on the shuffles of the higher components of the $A_{\infty}$ map $A \rightarrow \mathcal{A}$ ). Hence the terms of the antisymmetrization of (5) which do not show up in (7), sum up to zero, as required. 


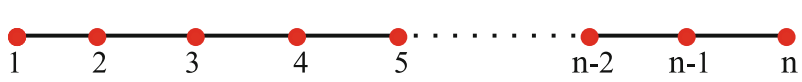

Fig. 2 Linear graph $G$

Example 17 Suppose that $G$ is a linear graph as in Fig. 2. Suppose also that $a_{1}, \ldots, a_{n} \in A$ are such that $m_{i}\left(a_{p}, \ldots, a_{p+1-i}\right)=0$ for all $i \geq 2$ and $1 \leq$ $p \leq k-i+1$. Then by corollary to Proposition 11 and Proposition 14 the product $a_{1} \otimes \cdots \otimes a_{n}$ represents a class in $E_{n-1}$ and $\partial_{n-1}\left(a_{1} \otimes \cdots \otimes a_{n}\right)$ is represented, up to sign, by $m_{n}\left(a_{1}, \ldots, a_{n}\right)$. Observe that by $[9,12]$ under the same assumptions the $n$-fold Massey product of $a_{1}, \ldots, a_{n}$ is well defined, and represented (as a coset), up to a sign, by the value $m_{n}\left(a_{1}, \ldots, a_{n}\right)$. Thus, considering sub-paths in a complete graph on $n$ vertices we give a concrete formulation (and proof) of the conjecture at the bottom of page 429 of [2] that "the higher differentials... are determined by higher-order Massey products". The $n=3$ case of this observation was proved earlier in [5].

\subsection{Degeneration}

Proposition 18 Assume that for a choice of homotopy $h$ all higher $A_{\infty}$ products van$i s h \mu_{i}=0, i \geq 3$ (e.g. $M$ is Kahler). Then the spectral sequence degenerates at the $E_{2}$ term: $\partial_{t}=0$ for $t \geq 2$. In general, if $k \leq n-1$ is the maximal length of a sub-tree in $G$, then the spectral sequence degenerates at the $E_{k+1}$ term: $\partial_{t}=0$ for $t \geq k+1$.

Proof For the first part, by homological perturbation theory, there is an $A_{\infty}$-map $A \rightarrow \mathcal{A}$ which induces a quasi-isomorphism of $A_{\infty}$-algebras. This can be encoded by a single $R$-linear map $B(A) \rightarrow \mathcal{A}$ such that the canonical multiplicative extension $\Omega(B(A)) \rightarrow \mathcal{A}$ is a quasi-isomorphism of $D G$-algebras, where $\Omega$ and $B$ are the cobar and bar constructions, respectively. But since the higher products vanish, the natural map $\Omega(B(A)) \rightarrow A$ is also a quasi-isomorphism of DG-algebras. Since the differential on $A$ is zero, the spectral sequence of $C_{A}(G)$ degenerates at the $E_{2}$ term.

For the second part, assume that $x \in E_{1}^{p, q}$ represents a class in $E_{k}^{p, q}$ and let us show that $\partial_{t}(x)=0$ for $t>k$. We can assume that $x$ is a linear combination of elements in $e_{s} \cdot A^{\otimes l(s)}$ with $|s|=p$ and fixed $l(s)=l$. Since $x$ represents an element in $E_{k}$, the system of equations (4) on $x_{\in} E_{1}^{p+j-1, q+1-j}$ with $j=2, \ldots, k-1$, admits a solution. From our results on the operators $d_{i}$ in the previous subsection, we can assume that $x_{j}$ is a linear combination of terms $e_{s} \cdot A^{\otimes l(s)}$ where $s$ contains a subtree of length $j-1$. By the same result $d_{i}\left(x_{j}\right)=0$ if $i+j-1>k$. Therefore $\partial_{t}(x)=0$ for $t>k$.

Remark 19 When $G$ is a complete graph on $n$ vertices, the maximal subtree length is $k=n-1$. Our result $\partial_{t}=0$ for $t \geq n$ is a little weaker than Proposition 4.2 in [5] which asserts that $\partial_{n-1}=0$ as well.

Acknowledgments V. Baranovsky wants to thank HSE in Moscow, Russia and USTC in Hefei, China where he stayed while working on this paper, for their excellent research conditions. R. Sazdanovic wants to thank the organizers of the Special Program "Homology theories for knots and links" for the opportunity to do research at MSRI where this collaboration started. 


\section{References}

1. Anick, D.J.: Hopf algebras up to homotopy. J. Am. Math. Soc. 2(3), 417-453 (1989)

2. Bendersky, M., Gitler, S.: The cohomology of certain function spaces.. Trans. AMS 326(1), 423-440 (1991)

3. Cheng, X.Z., Getzler, E.: Transferring homotopy commutative algebraic structures, J. Pure Appl. Algebra. 212, 2535-2542 (2008)

4. Estwood, M., Hugget, S.: Euler characteristics and chromatic polynomials. Eur. J. Comb. 28(6), 1553-1560 (2007)

5. Felix, Y., Thomas, J.-C.: Configuration spaces and Massey products. Int. Math. Res. Notices 33, 1685-1702 (2004)

6. Gelfand, I., Manin, Y.: Methods of homological algebra. Springer, Berlin (1996)

7. Helme-Guizon, L., Przytycki, J.H., Rong, Y.: Torsion in graph homology. Fundam. Math. 190, 139-177 (2006)

8. Helme-Guizon, L., Rong, Y.: A categorification for the chromatic polynomial. Algebraic Geom. Topol. (AGT) 5, 1365-1388 (2005)

9. Johansson, L., Lambe, L.: Transferring algebra structures up to homology equivalence. Math. Scand. 89, 181-200 (2001)

10. Kadeishvili, T.V.: The algebraic structure in the homology of an $A(\infty)$-algebra. Soobshch. Akad. Nauk Gruzin. SSR 108(2), 249-252 (1982)

11. Lawrence, A.: Higher order compositions in the Adams spectral sequence. Bull. Am. Math. Soc. 76, 874-877 (1970)

12. Lu, D.-M., Palmieri, J.H., Wu, Q.-S., Zhang, J.J.: A-infinity structure on ext-algebras. J. Pure Appl. Algebra 213(11), 2017-2037 (2009)

13. May, J.P.: Matric Massey products. J. Algebra 12, 533-568 (1969)

14. McClure, J.E., Smith, J.H.: Multivariable cochain operations and little $n$-cubes. J. Am. Math. Soc. 16, 681-704 (2003)

15. Romero, A., Rubio, J., Sergergaert, F.: Computing spectral sequences. J. Symb. Comput. 41, 1059-1079 (2006)

16. Stasheff, J.: $H$-spaces from a homotopy point of view. In: Lecture Notes in Mathematics, vol. 161. Springer, Berlin (1970) 